\title{
Resource Allocation in Space-Division Multiplexing-Based Elastic Optical Networks
}

\author{
Silvana Trindade ${ }^{1}$, Nelson Luis Saldanha da Fonseca ${ }^{1}$ \\ ${ }^{1}$ Institute of Computing - State University of Campinas
} (UNICAMP)

silvanadlrc.ic.unicamp.br, nfonsecadic.unicamp.br

\begin{abstract}
This paper presents the work developed in the MSc. thesis entitled "Resource Allocation in Space-Division Multiplexing-Based Elastic Optical Networks" which explores the resource allocation problem in Space-Division Multiplexing-based Elastic Optical Networks (SDM-EON). The proposed algorithms jointly address spectrum fragmentation, modulation format, crosstalk, and energy consumption. Results demonstrate that our algorithms increase the acceptance of requests for lightpath establishment while handling energy consumption, spectrum fragmentation, energy consumption, load balance, and crosstalk.
\end{abstract}

Resumo. Este artigo resume a tese de mestrado entitulada "Resource Allocation in Space-Division Multiplexing-Based Elastic Optical Networks", que explora diferentes soluções para o problema de alocação de recursos em redes ópticas elásticas baseadas em multiplexação por divisão espacial. Os algoritmos propostos tratam de forma conjunta a fragmentação espectral, formato de modulação, crosstalk e consumo de energia. Resultados demonstraram que os algoritmos propostos são capazes de aumentar a aceitação de requições para estabelecimento de caminhos de luz ao mesmo tempo que trata da fragmentação espectral, consumo de energia, banceamento de carga, e crosstalk.

\section{Introduction}

The increasing demand for bandwidth can no longer be supported by Single-Mode Fiber (SMF) since its capacity will soon be exhausted. The Space-Division Multiplexing (SDM) technology has been incorporated in Elastic Optical Networks (EONs) to cope with the continuous growth of the Internet traffic and the capacity limitation of SMF. In SDMEON with Multi-Core Fibers (MCFs), the number of slots for allocation is $c$ times greater than that in a single-core fiber, where $c$ is the number of cores of the fiber. Nonetheless, if the optical spectrum (resources) is not properly allocated, the network will not be able to cope with the increasing demand for bandwidth. Thus, the motivation of this work is to capacitate the Internet to transport new and highly demanding applications. In line with that, the objective of the MSc thesis was to create effective mechanisms for resource allocation in SDM-EON.

There are several changes in resource allocation in SDM-EON. One of them is to deal with inter-core crosstalk interference in MCFs, generated by a power leak between 
neighboring cores, which demands careful establishment of lightpath to maintain the Quality of Transmission (QoT). Adjacent cores generate greater crosstalk due to the short physical distance between them; cores with a large number of neighboring cores can suffer from strong crosstalk.

In dynamic scenarios, lightpaths have different setup and teardown times, and diverse bandwidth requirements. In such scenarios, slots are allocated and deallocated dynamically, leading to a state of the spectrum in which there are slots available for allocation but these are scattered along the spectrum in a way that they cannot be allocated continuously and contiguously to a lightpath. Such state is called spectrum fragmentation.

These issues characterize the problem of resource allocation in SDM-EON and need to be addressed by the Routing, (Modulation Format), Core, and Spectrum Allocation (RCSA/RMCSA) algorithm, used to establish lightpaths. In SDM-EON networks, RCSA algorithms generally use the following steps to establish a new lightpath: route selection; calculation of the number of frequency slots to accommodate the requested bandwidth; verification of the availability of contiguous and continuous slots to allocate the request and the allocation of frequency slots in one or more cores.

The contributions of the thesis advance the state-of-the-art by proposing novel algorithms and architectures for resource allocation in SDM-EON. In contrast with most existing works, we jointly address the spectrum fragmentation, modulation format, crosstalk, and energy consumption in our algorithms. The proposed algorithms significantly improve the allocation of the spectrum and decrease the blocking of requests for lightpath establishment and produce a balance between energy consumption and resource usage. The proposed virtual network (VN) architecture sets apart by being able to work alongside with any algorithm, allowing dealing with multiple issues at once.

The main contributions of the thesis are: i) an RCSA algorithm that leverages deadline-driven batch requests, postponing the establishment of lightpath within certain limits in order to increase the energy efficiency and request acceptance, ii) four proactive algorithms to decrease the spectrum fragmentation in SDM-EON networks, which employ different techniques, iii) a reactive algorithm for spectrum defragmentation using an Unsupervised Machine Learning (UML) algorithm, and iv) an architecture using supervised learning and reinforcement learning techniques to identify the cause of problems and roll out a plan for providing solutions to them. These contributions are quite relevant for building a future optical Internet that will transport new bandwidth hunger bandwidth.

The following sections introduce the proposed algorithms.

\section{Batch Grooming}

Postponing a lightpath establishment allows the formation of groups of requests, called batches, with requests that arrived in a close span of time and with the same source and destination nodes. Batch grooming concept has already been used for spectrum allocation in WDM and EON networks with single-mode fibers [de Santi et al. 2013, Moura et al. 2014]. Each connection demands a certain transmission rate, number of bytes to be transmitted and have different deadlines for the transmission to be completed. A batch can be postponed until the closest time at which the deadline still can be reached.

Traditional RCSA algorithms cannot handle batch requests. We extended 


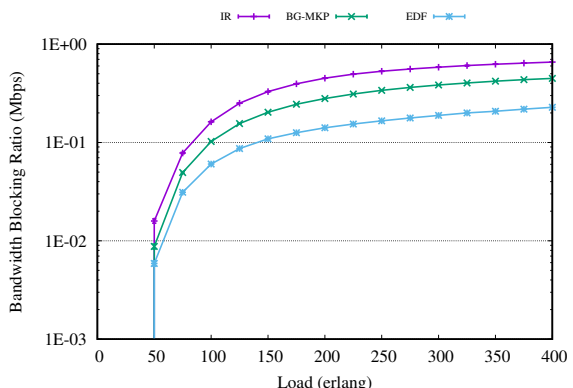

(a)

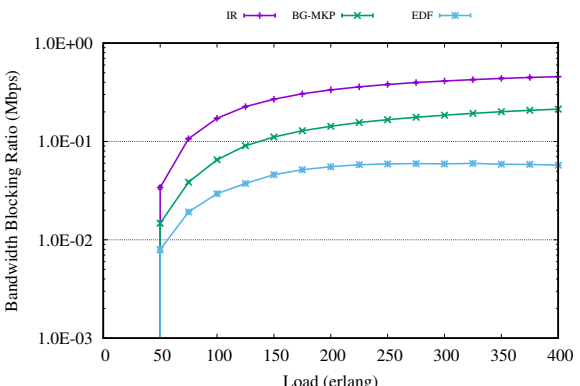

(b)

Figura 1. BBR as a function of the load for the NSFNET and USA topologies.

the Connected-Component Labeling (CCL) [Moura and da Fonseca 2016] and Inscribed Rectangle (IR) [Moura and da Fonseca 2017] RCSA algorithms to deal with allocation of batches of requests. We used the Multiple Knapsack Problem (MKP) to choose a set of slots in the spectrum. In the MKP formulation, blocks of slots are the knapsacks and the requests in a batch are the objects. The best block of slots to reserve for the requests is sought and slot waste is minimized to handle fragmentation.

For the evaluation of the algorithm we employed simulations, we used the NSFNET topology with 16 nodes and 25 fiber links and the USA topology with 24 nodes and 43 fiber links. Each fiber link is bidirectional and contains 7 cores, each with 320 slots and slot spacing of $12.5 \mathrm{GHz}$. The mean arrival rate and mean holding time were adjusted to simulate the desired load in erlangs.

The proposed solution produced lower Bandwidth Blocking Ratio (BBR) than did the traditional IR and CCL algorithms for the USA and the NSFNET topologies. Our algorithm produced BBR values up to one order of magnitude lower than that produced by the CCL algorithm, and up to $30 \%$ more energy efficiency. Figure 1 illustrates the $\mathrm{BBR}$ values for our algorithm with and without employing MKP, and the BBR given by the IR algorithm. Our algorithm produced BBR values $70 \%$ lower than the BBR values produced by the IR algorithm under loads of 200 erlangs.

\section{Spectrum Fragmentation}

When the spectrum is fragmented, resource allocation is greatly impaired. This is one key problem in resource allocation in SDM-EON. Several proposals have tried to address this problem. One [Tode and Hirota 2017] explores variations of the First-Fit (FF) policy, and uses a spectrum partitioning criterion to prevent crosstalk.

The work in [Yousefi and Rahbar 2018] adopts multipath routing to decrease blocking due to fragmentation. In [Xiong et al. 2019], the authors introduce an algorithm using supervised learning to predict traffic changes in order to avoid fragmentation. In [Zhang et al. 2014], a defragmentation algorithm was proposed to deal with the dynamic deallocation of the spectrum in SMFs.

The spectrum fragmentation problem can be tackled from many angles. The existing algorithms generally adapt algorithms created to handle the spectrum fragmentation in single-mode fibers. The problem with this approach is that it results in ineffective spectrum allocation since SDM-EON networks have over one core and suffer from inter-core 
crosstalk, which decreases the quality of transmission.

Proactive solutions try to reduce and prevent fragmentation occurrence by finding a route and a block of slots to allocate in a way that the probability of slot allocation for future requests increases. Our proactive algorithms consider the latency of the processing time. For the multi-path routing, we employ parallelism and use pre-computed orderings for spectrum allocation. Reactive solutions focus on spectrum defragmentation, which is the process of reducing fragmentation by reallocating and/or rerouting a set of existing lightpaths. Different from the literature, we explore the information that is generated in the defragmentation procedure to update dynamically an RMCSA algorithm.

Some of our proposed algorithms are fragmentation-aware (Fragmentationaware RMCSA (FA-RMCSA) and the FA Core prioritization RMCSA (FACPRMCSA) [Trindade and da Fonseca 2019]), different from the existing proposals that adopt route selection based purely on distance between the nodes. Instead, our algorithms use the closeness centrality measure and path fragmentation ratio to rank the candidate paths as criteria for route selection.

Our algorithms differ in the core and spectrum allocation step; the FA-RMCSA employs First-Core First-Fit (FCFF), while the FACP-RMCSA algorithm employs a core prioritization to avoid crosstalk. This core prioritization is unique since it provides an ordering which can reduce the occurrence of crosstalk.

A multipath routing algorithm for SDM-EON networks, called Split-Demand and Multipath Routing (SMR) algorithm, was introduced in the thesis. Different from existing solutions, the SMR algorithm considers constraints such as the number of slots and paths to establish multiple lightpaths. These constraints can help to evaluate the number of paths to split the requested lightpath. SMR uses parallelization in order to find slots available for allocation.

The SMR algorithm first tries to allocate a request using a single path, and only if that fails, the algorithm splits the bandwidth demand into multiple paths. As fragmentation increases, multipath routing is used more frequently. We conducted a study to find the best number of paths to split the bandwidth request to prevent further fragmentation of the spectrum. Results demonstrated that splitting requests with bandwidth higher than 100 $\mathrm{Gb}$ generated less spectrum fragmentation. Providing over three paths for the multipath routing tends to increase the fragmentation.

A proactive algorithm called Core and Quadrant Prioritization (CQP)-RMCSA algorithm was introduced to reduce the fragmentation of the spectrum by avoiding the generation of inter-cross crosstalk (core prioritization), and also by clustering the allocation of slots in sections of the spectrum (quadrant prioritization). The employment of these two criteria defines an order for slot allocation of arriving requests. We define this order offline. The only online overhead incurred is the search for available slots in an ordered list. By doing this, the flexibility for spectrum allocation is increased when compared to the existing algorithms, since, by using this ordering the occurrence of crosstalk and high fragmentation decrease. The routing selection finds a set of candidate paths given a source and destination pair. For the spectrum selection, the algorithm divides the set of frequency slots in all cores (spectrum) into four groups of slots called quadrants.

For the CQP-RMCSA, experiments were conducted to define the ideal number of 


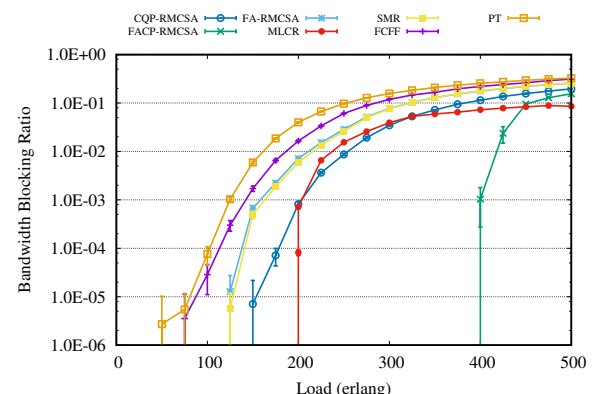

(a)

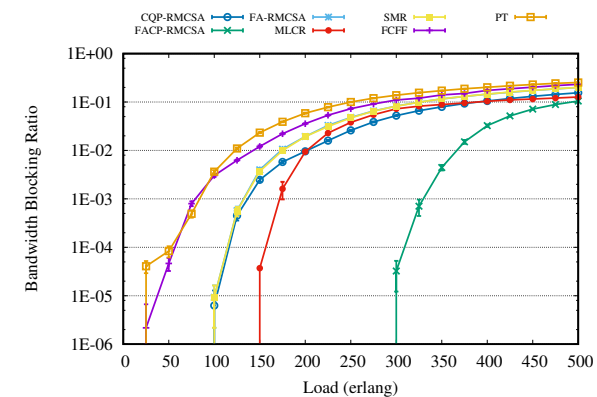

(b)

Figura 2. BBR as a function of the load for the NSFNET and USA topologies.

groups of slots to decrease the blocking probability. Results showed that four is the ideal number of groups and that higher number of groups would bring no additional benefit. It is preferable to allocate the maximum number of slots in one quadrant before allocating the slots in another quadrant. Since the quadrants are ordered, the allocation of slots must follow this order. The preferable core in a quadrant for slot allocation is the farthest to the others in that quadrant, considering the physical distance between the cores in the fiber.

A novel reactive algorithm using Unsupervised Machine Learning (ML), called ML Clusterized Reconfiguration (MLCR) algorithm was proposed to identify request patterns in real-time and adjust the spectrum occupancy to reduce fragmentation. The idea behind our defragmentation algorithm is to group requests by their characteristics, mapping clusters to cores and rearranging requests without performing rerouting. The unique point of the MLCR algorithm is the integration with the RMCSA algorithm realized by dynamically updating the latest information about the spectrum given by the UML algorithm after the defragmentation procedure. Such integration increases the probability of future requests to be accepted while decreasing the spectrum fragmentation.

Before the spectrum rearrangement, the MLCR algorithm maps clusters to cores. Initially, each cluster is mapped onto a core to guarantee that there are enough slots to allocate the requests being rearranged. This cluster mapping aims at leaving a large continuous and contiguous spectrum band to accept future requests for lightpath establishment. When the number of slots to allocate a cluster is not sufficient, the excess demand for slots can be mapped onto another core.

The proposed algorithms outperformed the existing algorithms. The FACPRMCSA algorithm started blocking requests only under loads of 400 erlangs for the NSFNET topology (Figure 2). The BBR values produced by the FA-RMCSA were three orders of magnitude lower than those produced by the PT algorithm under loads of 150 erlangs.

The BBR values produced by the CQP-RMCSA and SMR algorithms were three orders of magnitude lower when compared to the BBR produced by the PT algorithm under loads of 100 erlangs for the NSFNET topology (Figure 2).

Figure 2 illustrates that the SMR and FA-RMCSA algorithms produced better results compared to the FCFF and PT algorithms. The results produced by the SMR algorithm are due to the use of multiple paths, increasing the probability of lightpath 


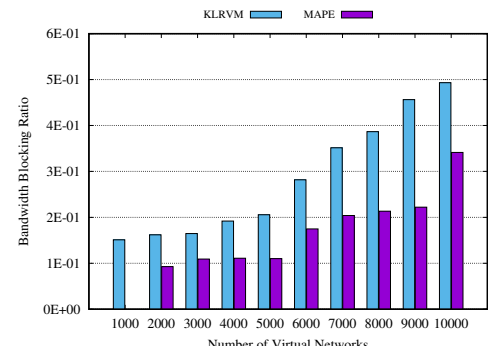

(a)

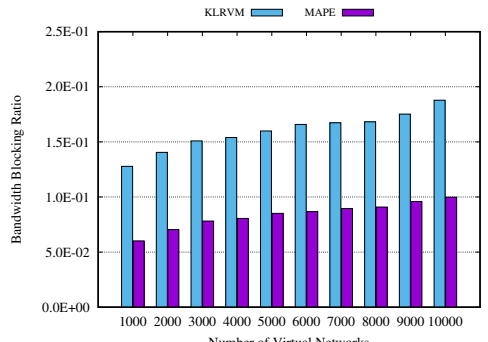

(b)

Figura 3. BBR as a function of the load for the NSFNET and CHNNET topologies.

establishing when the spectrum does not have a single block of contiguous and continuous slots in a path that satisfies the crosstalk and number of slots.

The BBR values produced by the MLCR algorithm were $40 \%$ lower than that produced by the PT algorithm under loads of 500 erlangs for USA topology. The MLCR algorithm significantly improves the amount of provisioned bandwidth compared to the PT and FCFF algorithms, producing the lowest BBR under loads of 400 erlangs, demonstrating it is very efficient under high loads compared to other algorithms. The MLCR algorithm extracts information correlated with the fragmentation problem, deciding of how to perform defragmentation more precisely. It also feeds the RCSA algorithm with information on the network state making a unique way to handle fragmentation.

\section{Network Virtualization}

Virtual networks (VNs) create an abstraction that simplifies the resource allocation and allows changes to be applied via software instead of needing changes to be done in the hardware when situations arise. In the thesis we leveraged virtualization to improve the resource allocation in SDM-EON by monitoring multiple issues at once, planning changes, testing them on the VNs and then applying them on the physical network.

The problem of the existing algorithms is that they focus on a single issue to tackle. The drawback is that there can be more than one source for poor network performance.

In the thesis, we propose an architecture, called cognitive Monitor, Analyze, Plan and Execute (MAPE) assistant, providing a new approach to assist resource allocation procedure to virtual networks. The MAPE comprises four functions that identifies a problem and plan a series of actions to reduce or eliminate it, using supervised and reinforcement learning algorithms. The monitor function pulls a set of metrics from the physical network and virtual topologies via the control plane in order to identify a potential problem. The problem identified is given to the analyze function to determine its criticality using a supervised learning algorithm. The analysis is then used by the plan function, which uses a reinforcement learning algorithm to generate an optimal plan to try to handle the existing problem. The execute function directs the execution plan for the control plane to execute, and then perform verifies to see if the changes were successful or not.

For the evaluation of the algorithm we employed simulations using a Poisson process, considering the NSFNET with 14 nodes and 21 fiber links and the CHNNET topology with 15 nodes and 27 fiber links. Using the MAPE algorithm, the VN acceptance ratio improved up to $25 \%$, the crosstalk decreased up to $15 \%$ and the BBR decreased 
up to $60 \%$ (Figure 3) compared to the Key-Link and Resources Contribution Degree for VNs Mappings (KLRVM) algorithm [Zhao et al. 2017]. One of the main advantages of our proposed algorithm is the fact that it can be used with any type of configuration algorithm, resulting in efficient use of resources. The MAPE algorithm introduced a new way to handle multiple issues in resource allocation to VNs. MAPE can be used with any VN configuration algorithm.

\section{Conclusion}

In the thesis, seven algorithms for resource allocation in SDM-EON networks were introduced, capable of handling different problems that can occur when this technology is used, mainly spectrum fragmentation, energy consumption, and crosstalk interference.

We used several metrics to analyze the performance of each algorithm, including the BBR, crosstalk, energy consumption, energy efficiency, network fragmentation ratio, Jain fairness index, $\mathrm{VN}$ acceptance ratio, link-load distribution, and average path length. Because of space limitation, in this paper, we showed only the BBR results, since it is the most common measure to evaluate the performance of algorithms for optical networks. Results were derived via simulation since there are very few private EON-SDM testbeds and would be too costly to develop one for this thesis. Current emulators, such as Mininet, are not adequate for such evaluation.

All algorithms introduced in the thesis significantly reduced the blocking of requests, increasing the efficiency of spectrum usage. Using batch requests, the energy efficiency was up to $30 \%$ higher when compared to the existing algorithms. The set of algorithms that aim at reducing fragmentation achieved significant results, up to $60 \%$ lower BBR when compared to the existing ones. During the allocation process, these algorithms were capable of handling spectrum fragmentation more gracefully. Crosstalk interference was also reduced by employing the proposed algorithms. By using MAPE, the crosstalk values were decreased by up to $15 \%$, increasing the QoT.

The BBR values results differ depending on the topology because of the connectivity degree. Since the USA and CHNNET topologies have higher connectivity degree than does NSFNET topology, they exhibit lower BBR values. Indeed, the connectivity degree is inversely correlated with the BBR.

For future work, we intend to explore different learning algorithms to improve resource usage, requiring a large dataset from the real-world, which was not available at the time. We also intend to explore other technologies, such as multi/few-mode fibers, and other problems, like survivability and sustainable networks.

\section{Publications}

- Silvana Trindade and Nelson L. S. da Fonseca. Proactive Fragmentation-aware Routing, Modulation Format, Core, and Spectrum Allocation in SDM-EON. 53rd IEEE International Conference on Communications (ICC), 2019 (A1, H-index = 61).

- Silvana Trindade and Nelson L. S. da Fonseca. Core and Spectrum Allocation for Avoidance of Spectrum Fragmentation in SDM-EON. 54rd IEEE International Conference on Communications (ICC), 2020 (A1, H-index =61). Accepted for publication. 
- Silvana Trindade and Nelson L. S. da Fonseca. $K$-Means Clustering for Spectrum Defragmentation in Space-Division Multiplexing Elastic Optical Networks. IEEE Communication Magazine (JCR = 10.3). Submitted.

- Silvana Trindade and Nelson L. S. da Fonseca. Deadline-Driven Batch Requests in Elastic Optical Networks with Space-Division Multiplexing. IEEE Global Communications Conference, 2020 (A1, H-index = 91). Submitted.

Moreover, two manuscripts are at the final stage for submissions to journals:

- Silvana Trindade and Nelson L. S. da Fonseca. Spectrum Fragmentation Problem: A Comparison of Different Proactive Solutions in SDM-EON.

- Silvana Trindade and Nelson L. S. da Fonseca. Cognitive Assistant for Elastic Optical Networks Space-Division Multiplexing.

\section{Referências}

de Santi, J., da Fonseca, N. L., and Figueiredo, G. B. (2013). Algorithm for traffic grooming of batches of deadline-driven requests. In Communications (ICC), 2013 IEEE International Conference on, pages 2267-2271. IEEE.

Moura, P. M. and da Fonseca, N. L. (2016). Routing, core and spectrum assignment based on connected component labelling for sdm optical networks. In Communications (ICC), 2016 IEEE International Conference on, pages 1-6. IEEE.

Moura, P. M. and da Fonseca, N. L. (2017). Inscribed rectangles algorithm for routing, core and spectrum assignment for sdm optical networks. In Communications (ICC), 2017 IEEE International Conference on, pages 1-6. IEEE.

Moura, P. M., da Fonseca, N. L., and Scaraficci, R. A. (2014). Traffic grooming of batches of deadline-driven requests in elastic optical networks. In Global Communications Conference (GLOBECOM), 2014 IEEE, pages 1284-1289. IEEE.

Tode, H. and Hirota, Y. (2017). Routing, spectrum, and core and/or mode assignment on space-division multiplexing optical networks. Journal of Optical Communications and Networking, 9(1):A99-A113.

Trindade, S. and da Fonseca, N. L. S. (2019). Proactive fragmentation-aware routing, modulation format, core, and spectrum allocation in eon-sdm. In ICC 2019 - 2019 IEEE International Conference on Communications (ICC), pages 1-6.

Xiong, Y., Yang, Y., Ye, Y., and Rouskas, G. N. (2019). A machine learning approach to mitigating fragmentation and crosstalk in space division multiplexing elastic optical networks. Optical Fiber Technology, 50:99-107.

Yousefi, F. and Rahbar, A. G. (2018). Novel fragmentation-aware algorithms for multipath routing and spectrum assignment in elastic optical networks-space division multiplexing (eon-sdm). Optical Fiber Technology, 46:287-296.

Zhang, M., You, C., Jiang, H., and Zhu, Z. (2014). Dynamic and adaptive bandwidth defragmentation in spectrum-sliced elastic optical networks with time-varying traffic. Journal of Lightwave Technology, 32(5):1014-1023.

Zhao, G., Xu, Z., Ye, Z., Wang, K., and Wu, J. (2017). A load balancing algorithm based on key-link and resources contribution degree for virtual optical networks mapping. In Computer, Information and Telecommunication Systems (CITS), 2017 International Conference on, pages 63-67. IEEE. 